\title{
Lifetime of an emitting dipole near various types of interfaces including magnetic and negative refractive materials
}

\author{
Raphael Ruppin ${ }^{\text {a) }}$ and Olivier J. F. Martin \\ Nanophotonics and Metrology Laboratory, Swiss Federal Institute of Technology Lausanne (EPFL), \\ 1015 Lausanne, Switzerland
}

(Received 3 August 2004; accepted 14 September 2004)

\begin{abstract}
A classical electromagnetic calculation of the lifetime of an emitting electric dipole near a material slab is presented. The lifetime is deduced from the imaginary part of the electric field Green's tensor associated with the stratified medium. The method is applied not only to the well known case of metallic reflectors, but also to magnetic reflectors and to negative refractive index slabs. The frequency dependence of the nonradiative decay rate at small distances is analyzed and interpreted in terms of the surface polariton modes of the slab. (C) 2004 American Institute of Physics.
\end{abstract}

[DOI: $10.1063 / 1.1812742]$

\section{INTRODUCTION}

The modification of molecular fluorescence near a metal surface has been the subject of many experimental and theoretical investigations. ${ }^{1-10}$ It was found that for distances of the order of a few wavelengths from the metal surface the fluorescence lifetime oscillated as a function of distance, while for small distances the lifetime decreased monotonically towards zero. The oscillations result from interference between the emitted radiation and the wave reflected from the metallic surface. The decrease in the lifetime at small distances is due to nonradiative energy transfer into the metal. These phenomena can be derived theoretically from the model of an oscillating point dipole near a surface. This model was already treated by Sommerfeld in his investigation of the radio frequency radiation of a radiating dipole above the conducting earth. ${ }^{11}$ Quantum mechanical calculations of the coupling of excited molecules to the surface have also been performed, and it was found that the results are similar to those derived by classical electromagnetic theory. ${ }^{5,8}$ The dependence of the nonradiative energy transfer rate on frequency has been analyzed, and the peaks have been interpreted in terms of the relevant surface plasmon modes of the metal. ${ }^{4,5,7,8,10}$

In all of the works mentioned above, the reflecting surface was metallic. The question arises whether a magnetic, nonmetallic reflector will exhibit similar effects. Whereas the metallic reflector has a dielectric permittivity with a negative real part, the magnetic reflector is characterized by a magnetic permeability with a negative real part. The possibility of engineering artificial materials of this type has recently been demonstrated. ${ }^{12,13}$ Here we employ the Green's tensor technique to the calculation of the lifetime and nonradiative decay rate of a dipole near a surface. We compare the cases of metallic and magnetic reflectors, and also treat the case of a dipole near the surface of a negative index material. Although the frequency range of presently available metamaterials with negative permeability is in the microwave region,



the goal of extending their range to higher frequencies might be achievable in the future.

The results presented here are also useful at microwave frequencies, since there are close similarities between the radiation of a fluorescent molecule and that of a dipolar antenna. For the latter, the field reflected by the surface disturbs the antenna emission, leading to an increased antenna radiation resistance, the real part of the impedance. In a more general way, the following results can be applied to any radiating system where dipolar interaction plays a dominant role, such as in quantum dots. ${ }^{14}$

\section{LIFETIME EVALUATION USING THE GREEN'S TENSOR}

The normalized lifetime $\tau$ of a dipolar source oriented in the $\alpha$ direction $(\alpha=x, y, z)$ in the presence of one or several material interfaces can be deduced from the imaginary part of the $\alpha \alpha$ component of the electric field Green's tensor G associated with the corresponding stratified medium. ${ }^{15,16}$ Using the same unit system as in Ref. 17, we obtain

$$
\tau=\left(1+\frac{4 \pi}{k} \operatorname{Im}\left\{\mathbf{G}_{\alpha \alpha}\right\}\right)^{-1}
$$

where $k$ is the wave number of the medium in which the dipole is located. The lifetime in Eq. (1) is normalized to its value in an infinite homogeneous medium with wave number $k$. The technique described in Ref. 18 is used to compute the Green's tensor associated with a stratified medium composed of material layers with arbitrary permittivity $\epsilon_{i}$ and permeability $\mu_{i}$. This approach consists in expanding the field of an electric dipole in plane waves and summing up all the corresponding reflected and refracted components at the different material interfaces in the stratified medium. The corresponding Sommerfeld integral is computed by deforming the integration path in the complex plane, as described in Ref. 18. It should be emphasized here that difficulties arise when a material layer has $\epsilon=\mu=-1$. In that lossless case, the excitation of surface waves with infinite quality factor $Q$ on the material interface leads to a singular behavior of the 


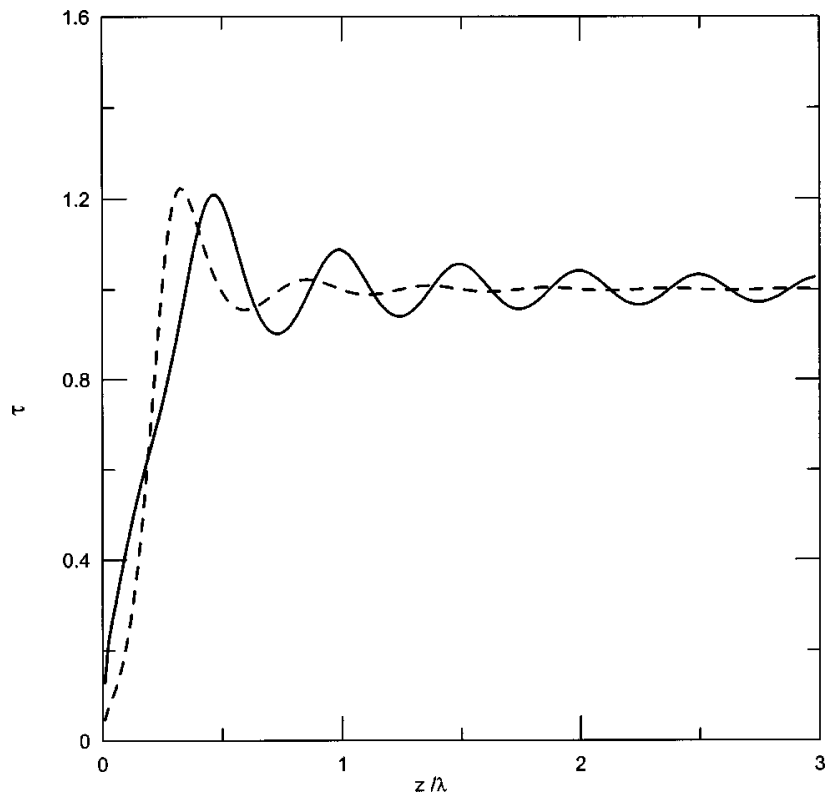

FIG. 1. Normalized lifetime of dipole near a slab of thickness $20 \lambda$, with $\epsilon=-3+0.01 i$ and $\mu=3+0.01 i$. Full curve-dipole parallel to surface, broken curve-dipole perpendicular to surface.

electric field, which renders the numerical problem intractable. ${ }^{19}$ However, in practical realization of negative refraction materials, some losses are always present, including in the microwave regime. ${ }^{12,13}$ These losses make the numerical problem well behaved and the integration technique presented in Ref. 18 can be applied straightforwardly.

\section{NUMERICAL RESULTS AND DISCUSSION}

We apply the Green's tensor technique to obtain the dipole lifetime for three types of surfaces: (a) $\operatorname{Re}[\epsilon(\omega)]<0$ and $\operatorname{Re}[\mu(\omega)]>0$, as is the case for metals below the plasma frequency; (b) $\operatorname{Re}[\epsilon(\omega)]>0$ and $\operatorname{Re}[\mu(\omega)]<0$, which is achievable with magnetically dispersive, nonmetallic materials; (c) $\operatorname{Re}[\epsilon(\omega)]<0$ and $\operatorname{Re}[\mu(\omega)]<0$, i.e., a negative refractive index material.

The calculations are performed for a slab of thickness $d=20 \lambda$, where $\lambda$ is the wavelength at the dipole frequency. The dipole is in vacuum, at a distance $z$ from the surface of the slab. The results of the normalized lifetime calculations for a metallic slab with negative $\operatorname{Re}[\epsilon(\omega)]$ and positive $\operatorname{Re}[\mu(\omega)]$ are shown in Fig. 1. The well-known oscillatory behavior due to interference effects is obtained. The amplitude of the oscillations increases with decreasing $z$, but at very small distances from the surface, the lifetime decreases sharply due to nonradiative energy transfer into the slab.

We now interchange the values of the permittivity and the permeability, so that we have a nonmetallic slab, with negative real part of the permeability. The results for this case, shown in Fig. 2, again exhibit the oscillations which decrease at large distances, and the sharp nonradiative decrease of the lifetime at small distances. The main difference, compared with Fig. 1, is that the nonradiative decay begins to prevail at smaller distances, so that higher lifetime oscillations are obtained. Another interesting difference is that in the interference pattern, the maxima of Fig. 2 appear where

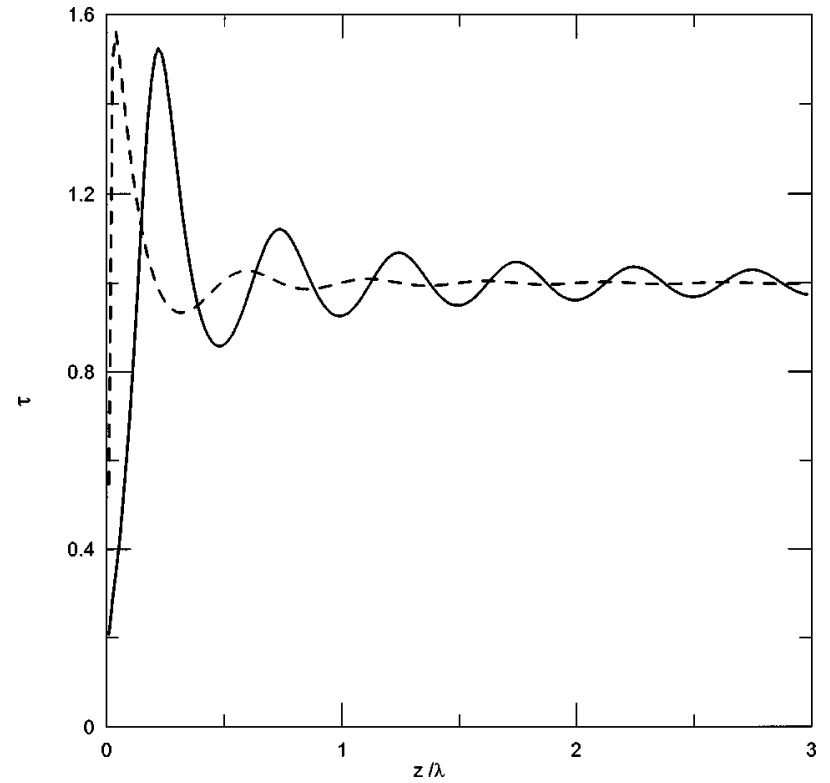

FIG. 2. Normalized lifetime of dipole near a slab of thickness $20 \lambda$, with $\epsilon$ $=3+0.01 i$ and $\mu=-3+0.01 i$. Full curve-dipole parallel to surface, broken curve-dipole perpendicular to surface.

in Fig. 1 the minima have occurred. In other words, there is a half wavelength shift between the two patterns. The origin of this shift can be traced to the phase shift of $\pi$ which occurs in the reflection coefficient when the values of $\epsilon$ and $\mu$ are interchanged. The results for the case of a negative index material are shown in Fig. 3. As expected, the interference pattern practically disappears, because the electromagnetic radiation can propagate through the slab, which has the same impedance as the vacuum where the dipole is located.

For the case of metals, the decrease of the dipole lifetime at very short distances has been interpreted in terms of the excitation of surface plasmons. The decay rate of the dipole

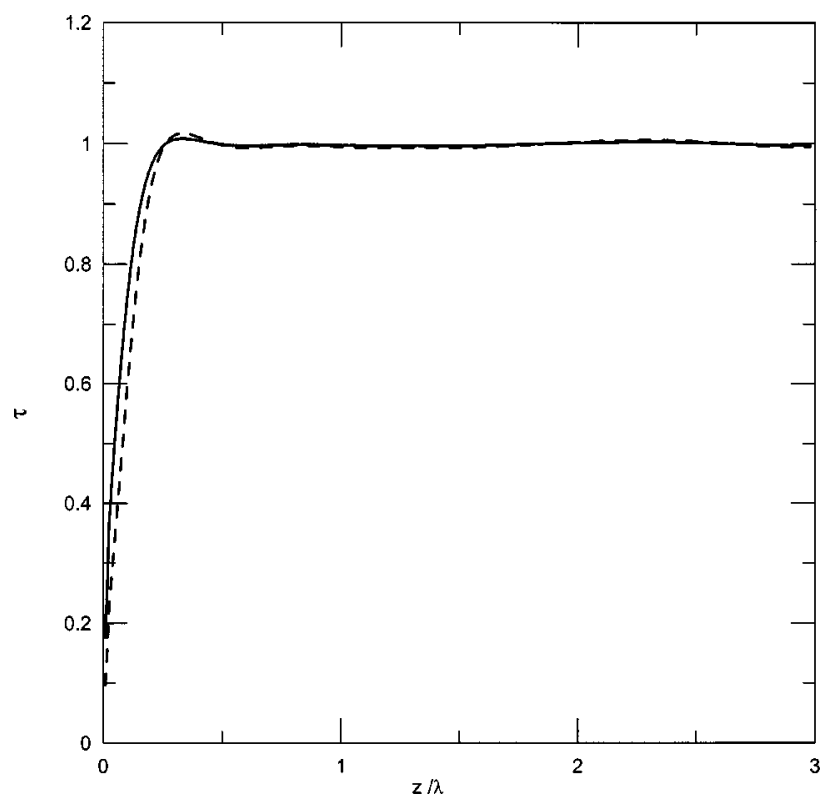

FIG. 3. Normalized lifetime of dipole near a slab of thickness $20 \lambda$, with $\epsilon$ $=-3+0.01 i$ and $\mu=-3+0.01 i$. Full curve-dipole parallel to surface, broken curve-dipole perpendicular to surface. 


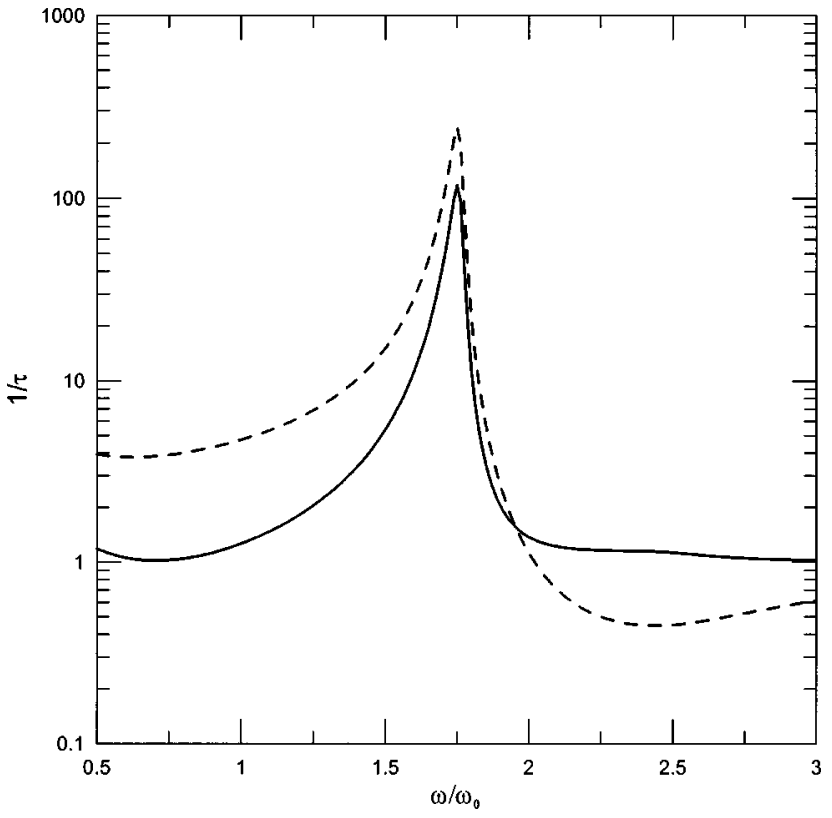

FIG. 4. Frequency dependence of normalized decay rate of a dipole at a distance of $0.03 \lambda_{0}$ from a slab of thickness $10 \lambda_{0}$. The slab permittivity is of the form (2). Full curve-dipole parallel to surface, broken curve-dipole perpendicular to surface.

is a measure of the overlap between the near fields of the dipole and the fields of the surface plasmons of the slab. The slab surface plasmons are nonradiative and cannot be excited directly by a propagating incident electromagnetic plane wave. The near field of the dipole has evanescent components, and can therefore excite the surface plasmons. In order to interpret the nonradiative decay in terms of the various surface modes of the slab, we calculate its frequency dependence. Negative values of either the permittivity or the permeability necessarily mean that the material is dispersive. For our calculations we assume the following typical forms for the frequency dependence of slab permittivity and permeability:

$$
\begin{aligned}
& \epsilon(\omega)=1-\frac{\omega_{p}^{2}}{\omega(\omega+i \gamma)}, \\
& \mu(\omega)=1-\frac{F \omega_{0}^{2}}{\omega^{2}-\omega_{0}^{2}+i \Gamma \omega} .
\end{aligned}
$$

Here, $\omega_{p}$ is an effective plasma frequency, $\omega_{0}$ is the resonance frequency of the permeability, and $\gamma$ and $\Gamma$ are damping frequencies. In the numerical calculations the following parameters are used: $\omega_{p}=2.5 \omega_{0}, \gamma=0.01 \omega_{p}, \Gamma=0.01 \omega_{0}$, and $F=1.25$. We calculate the normalized decay rate (inverse of normalized lifetime) for a dipole located close to the slab surface, at a distance of $z=0.03 \lambda_{0}$, where $\lambda_{0}$ is the free space wavelength corresponding to the frequency $\omega_{0}$. The slab is assumed to be thick with $d=10 \lambda_{0}$. First, we check the case of no magnetic dispersion, i.e., $\mu(\omega)=1$. The calculated energy transfer rate is shown in Fig. 4. As expected, the energy transfer rate peaks at the surface plasmon frequency, defined as the root of the equation $\epsilon(\omega)=-1$, which yields the value $\omega / \omega_{0}=1.77$. This metallic type surface plasmon is

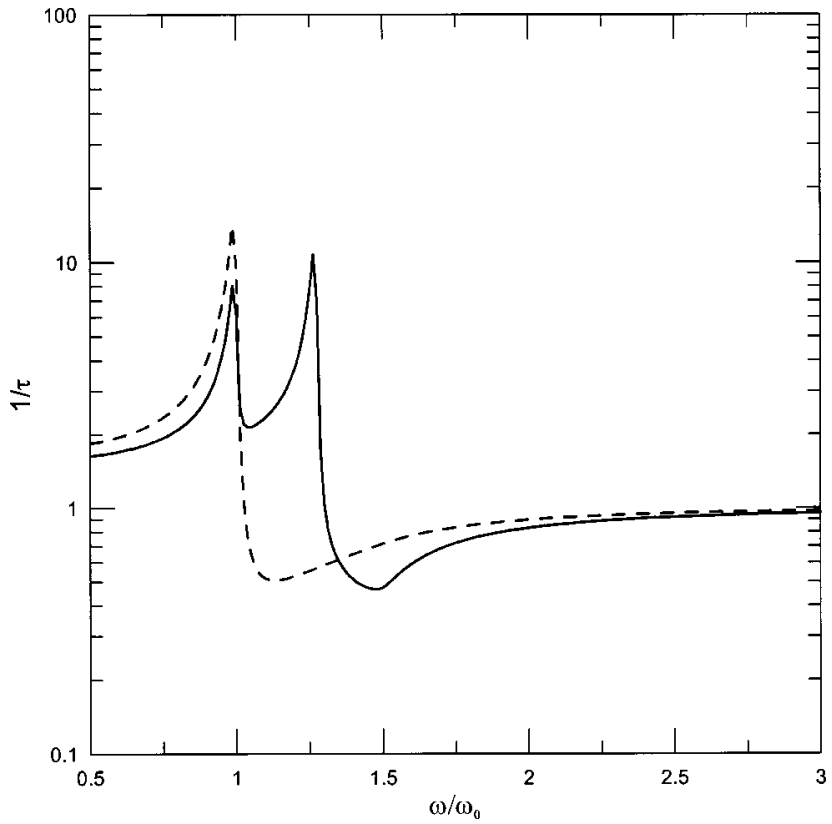

FIG. 5. Frequency dependence of normalized decay rate of a dipole at a distance of $0.03 \lambda_{0}$ from a slab of thickness $10 \lambda_{0}$. The slab permeability is of the form (3). Full curve-dipole parallel to surface, broken curve-dipole perpendicular to surface.

$p$ polarized. Thus, its electric field has components both parallel and perpendicular to the slab. Therefore it is excited by dipoles of both polarizations. Next we examine the case of no dielectric dispersion, i.e., $\epsilon(\omega)=1$, and the results are shown in Fig. 5. The peak at $\omega_{0}$ is due to a $p$ polarized magnetic polariton. The higher frequency peak is due to the $s$ polarized surface mode branch, which converges at large wave vectors to the frequency given by the root of the equation $^{20} \mu(\omega)=-1$. For the parameters employed here,

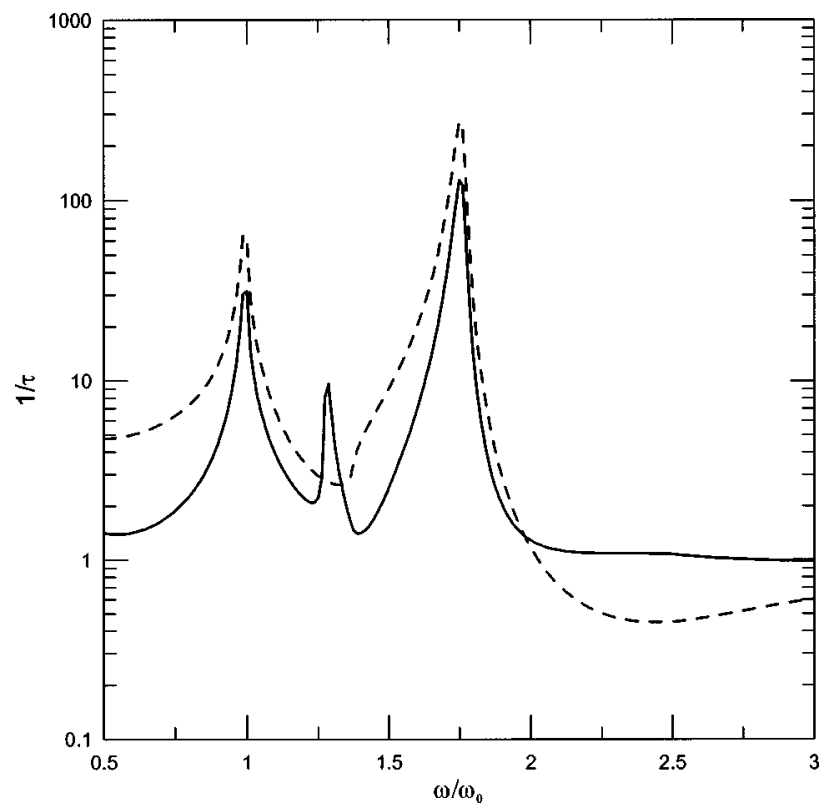

FIG. 6. Frequency dependence of normalized decay rate of a dipole at a distance of $0.03 \lambda_{0}$ from a slab of thickness $10 \lambda_{0}$. The slab permittivity is of the form (2) and the permeability of the form (3). Full curve-dipole parallel to surface, broken curve-dipole perpendicular to surface. 


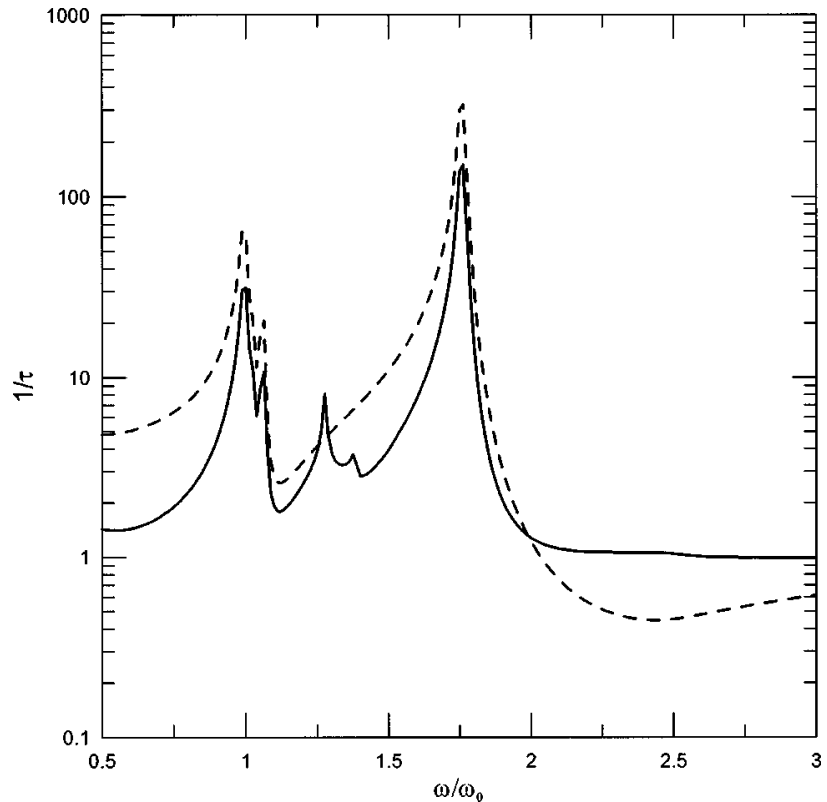

FIG. 7. Frequency dependence of normalized decay rate of a dipole at a distance of $0.03 \lambda_{0}$ from a slab of thickness $0.1 \lambda_{0}$. The slab permittivity is of the form (2) and the permeability of the form (3). Full curve-dipole parallel to surface, broken curve-dipole perpendicular to surface.

this yields the value $\omega / \omega_{0}=1.28$, and for the parallel dipole a peak indeed occurs at this frequency. The perpendicular dipole cannot excite an $s$ polarized surface mode. The calculated frequency dependence of the decay rate, when the slab has both dielectric and magnetic dispersion, is shown in Fig. 6. It is found that all peaks shown in Figs. 4 and 5 appear, the $p$ polarized ones for any polarization of the dipole, and the $s$ polarized one only for the dipole which is parallel to the surface. So far, the calculations were performed for a thick slab. For a thin slab with $d=0.1 \lambda_{0}$ the spectral dependence of the decay rate assumes the form shown in Fig. 7. The three surface polariton peaks again appear, and two subsidiary peaks are found to occur. The subsidiary peak at $\omega / \omega_{0}$ $=1.06$ is outside the frequency region of the slab surface polaritons. ${ }^{20}$ We therefore assign it to a waveguide mode, i.e., a mode having an oscillatory behavior inside the slab and an evanescent behavior outside it. The weak peak at $\omega / \omega_{0}=1.37$ results from the splitting of the $s$ polarized surface polariton, due to the interaction between the surface modes localized at the two surfaces. The analogous effect in metals, of the coupling of a dipole to plasmons localized near the two surfaces of a thin silver film has recently been reported. ${ }^{10}$
In conclusion, we have calculated the lifetime of an emitting dipole near three types of material slabs: (1) Metallic slab, which is the case studied extensively in the past, (2) magnetic reflector slab, possessing a negative real part of the magnetic permeability, and (3) negative refractive index material slab, with negative real part of both the permittivity and the permeability. We have found that for the magnetic reflector, the dependence of the dipole lifetime on the separation of the dipole from the surface is qualitatively similar to that previously found for a metallic reflector. The lifetime oscillates as the distance of the dipole from the surface is varied, and it decreases sharply at very short distances, due to nonradiative decay. For a negative refractive index material the oscillations are hardly observable, because the radiation emitted from the dipole can propagate through the slab, so that interference effects are minimal. Using typical models for the dispersion of the permittivity and the permeability, we have analyzed the frequency dependence of the decay rate at small distances in terms of the surface modes of the slab. While in previous studies only peaks due to $p$ polarized surface polaritons were predicted, for a magnetic reflector, or for a negative index material, $s$ polarized surface mode peaks also appear.

${ }^{1}$ K. H. Drexhage, J. Lumin. 1/2, 693 (1970).

${ }^{2}$ K. H. Tews, J. Lumin. 9, 223 (1974).

${ }^{3}$ R. R. Chance, A. Prock, and R. Silbey, J. Chem. Phys. 60, 2744 (1974).

${ }^{4}$ H. Morawitz and M. R. Philpott, Phys. Rev. B 10, 4863 (1974).

${ }^{5}$ M. R. Philpott, J. Chem. Phys. 62, 1812 (1975).

${ }^{6}$ R. R. Chance, A. H. Miller, A. Prock, and R. Silbey, J. Chem. Phys. 63, 1589 (1975)

${ }^{7}$ R. R. Chance, A. Prock, and R. Silbey, Adv. Chem. Phys. 37, 1 (1978).

${ }^{8}$ J. M. Wylie and J. E. Sipe, Phys. Rev. A 30, 1185 (1984).

${ }^{9}$ G. W. Ford and W. H. Weber, Phys. Rep. 113, 195 (1984).

${ }^{10}$ R. M. Amos and W. L. Barnes, Phys. Rev. B 55, 7249 (1997).

${ }^{11}$ A. Sommerfeld, Ann. Phys. (Leipzig) 28, 665 (1909).

${ }^{12}$ R. Shelby, D. R. Smith, and S. Schultz, Science 292, 77 (2001).

${ }^{13}$ C. G. Parazzoli, R. B. Greegor, K. Li, B. E. C. Koltenbah, and M. Tanielian, Phys. Rev. Lett. 90, 107401 (2003).

${ }^{14}$ T. Kawazoe, K. Kobayashi, S. Sangu, and M. Ohtsu, Appl. Phys. Lett. 82, 2957 (2003).

${ }^{15}$ E. N. Economu, Green's Functions in Quantum Physics (Springer, Berlin, 1983).

${ }^{16}$ F. Wijnands, J. B. Pendry, F. J. Garcia-Vidal, P. M. Bell, P. J. Roberts, and L. Martin Moreno, Opt. Quantum Electron. 29, 199 (1997).

${ }^{17}$ O. J. F. Martin and N. Piller, Phys. Rev. E 58, 3909 (1998).

${ }^{18}$ M. Paulus, P. Gay-Balmaz, and O. J. F. Martin, Phys. Rev. E 62, 5797 (2000).

${ }^{19}$ W. C. Chew, Microwave Opt. Technol. Lett. 42, 369 (2004).

${ }^{20}$ R. Ruppin, J. Phys.: Condens. Matter 13, 1811 (2001). 